\title{
Efficient and Highly Aldehyde Selective Wacker Oxidation
}

2012

Vol. 14, No. 13

3237-3239

\author{
Peili Teo, ${ }^{\dagger, \ddagger}$ Zachary K. Wickens, ${ }^{\dagger}$ Guangbin Dong, ${ }^{\S}$ and Robert H. Grubbs ${ }^{\star, \dagger}$ \\ Arnold and Mabel Beckman Laboratory of Chemical Synthesis, Division of Chemistry \\ and Chemical Engineering, California Institute of Technology, Pasadena, California \\ 91125, United States, Institute of Chemical and Engineering Sciences, 1 Pesek Road, \\ Jurong Island, 627833, Singapore, and Department of Chemistry and Biochemistry, \\ The University of Texas at Austin, 1 University Station A5300, Austin, Texas 78712, \\ United States
}

rhg@caltech.edu

Received May 17, 2012

ABSTRACT

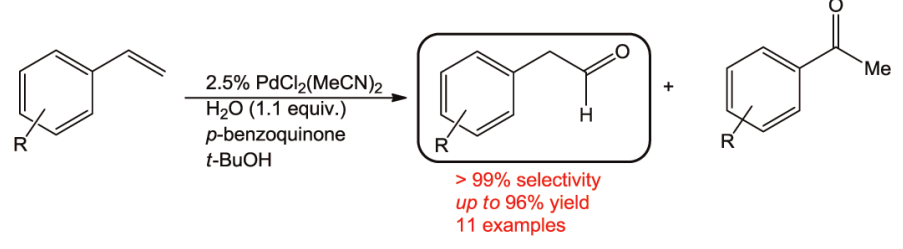

A method for efficient and aldehyde-selective Wacker oxidation of aryl-substituted olefins using $\mathrm{PdCl}_{2}(\mathrm{MeCN})_{2}, 1,4-$-benzoquinone, and $t$-BuOH in air is described. Up to a $96 \%$ yield of aldehyde can be obtained, and up to $99 \%$ selectivity can be achieved with styrene-related substrates.

The $\mathrm{Pd}^{\mathrm{II}}$-catalyzed oxidation of olefins to carbonyl compounds, commonly known as the Wacker oxidation, is one of the most well-known Pd mediated reactions and has extensive synthetic applications. The Wacker oxidation, however, follows Markonikov's rule and thus yields methyl ketones from the majority of terminal olefins (see reaction in abstract). The Wacker oxidation has been broadly adopted by synthetic chemists, to the degree that terminal olefins are often viewed as masked ketones. ${ }^{1,2}$ In specific cases, aldehyde selectivity has been observed, albeit usually in low yields. However, these examples are restricted to cases where either the olefin is functionalized with a directing group ${ }^{3-5}$ or stoichiometric palladium or excess heteropolyacid is employed. ${ }^{2,6}$ Recently, the

\footnotetext{
California Institute of Technology.

Institute of Chemical and Engineering Sciences.

$\S$ The University of Texas at Austin.

(1) Tsuji, J. Palladium Reagents and Catalysts-New Perspectives for the 21st Century; J. Wiley \& Sons Ltd.: U.K., 2004.

(2) Muzart, J. Tetrahedron 2007, 63, 7505.

(3) Wenzel, T. T. J. Chem. Soc., Chem. Commun. 1993, 862.

(4) Weiner, B.; Baeza, A.; Jerphagnon, T.; Feringa, B. L. J. Am. Chem. Soc. 2009, 131, 9473.

(5) Michel, B. W.; McCombs, J. R.; Winkler, A.; Sigman, M. S. Angew. Chem., Int. Ed. 2010, 49, 7312.

(6) Wright, J. A.; Gaunt, M. J.; Spencer, J. B. Chem.-Eur. J. 2006, 12,949 .
}

synthesis of terminal acetals via palladium catalyzed addition of pinacol was reported $;{ }^{7}$ however there is still a need for the direct synthesis of aldehyde. Current methods for aldehyde production from carbon-carbon multiple bonds are hydroformylation and alkyne hydration. However, hydroformylation produces the homologous aldehyde and anti-Markovnikov alkyne hydration often employs catalysts that are nontrivial to prepare. ${ }^{8-10}$ Another popular method for aldehyde production is via alcohol oxidation, but the formation of primary alcohols can itself be a difficult process. ${ }^{11,12}$

Hence, there is a need to develop a simple yet efficient process to convert olefins directly into aldehydes with high yields and selectivity.

Although moderate aldehyde selectivities have been obtained for styrene Wacker oxidations, the report required either superstoichiometric heteropolyacids (HPA)

(7) Yamamoto, M.; Nakaoka, S.; Ura, Y.; Kataoka, Y. Chem. Commun. 2012, 48, 1165.

(8) Grotjahn, D. B.; Lev, D. A. J. Am. Chem. Soc. 2004, 126, 12232. 2867.

(9) Tokunaga, M.; Wakatsuki, Y. Angew. Chem., Int. Ed. 1998, 37,

(10) Hintermann, L.; Labonne, A. Synthesis 2007, 8, 1121.

(11) Beller, M.; Seayad, J.; Tillack, A.; Jiao, H. Angew. Chem., Int. Ed. 2004, 43, 3368 .

(12) Smith, M. B.; March, J. March's Advanced Organic Chemistry: John Wiley and Sons: New York, 2001. 
that contain 12 equiv of metal per mol of HPA or stoichiometric palladium. Above that, the reaction has to be carried out under an inert atmopshere. Hence, a practical method for aldehyde selective oxidation of styrene and structurally related olefins remains to be realized. ${ }^{1,2,6,13}$ The aldehyde products are important precursors to terminal alcohols ${ }^{13}$ as well as essential ingredients in artificial flavors, perfumery, and soaps. ${ }^{14,15}$ Herein, we report a new catalytic system that delivers high aldehyde selectivity of up to $>99 \%$ with high catalytic efficiencies without the need for an inert atmosphere.

During our development of a method for antiMarkonikov olefin hydration, we elaborated upon a strategy for the anti-Markonikov Wacker oxidation of olefins (reaction in abstract). ${ }^{13}$ The use of $t-\mathrm{BuOH}$ to deliver high aldehyde selectivities in Wacker oxidation has been well established, albeit often in low yields. ${ }^{2,3,16,17} p$-Benzoquinone (BQ) is widely used as a hydrogen acceptor and twoelectron oxidant in $\mathrm{Pd}^{\mathrm{II}}$-catalyzed reactions. ${ }^{18}$ By combining $\mathrm{PdCl}_{2}\left(\mathrm{MeCN}_{2}, \mathrm{BQ}\right.$, and $t-\mathrm{BuOH}$ in the presence of stoichiometric amounts of water, we found that both high aldehyde selectivity and high yields could be obtained with styrene as the substrate (Tables 1,2 ). The reaction with styrene is highly efficient: a TOF of $37 \mathrm{~h}^{-1}$ with $98 \%$ aldehyde selectivity could be obtained. Such a high TOF and selectivity is unprecedented for undirected aldehyde selective Wacker oxidations. Without BQ, no appreciable turnovers were observed. When the reaction is carried out in $i$-PrOH instead of $t-\mathrm{BuOH}$, the selectivity fell to $74 \%$ and the TOF was nearly halved (Table 1). Without adding $\mathrm{H}_{2} \mathrm{O}$, only a $38 \%$ yield could be obtained. We attribute this nonzero aldehyde yield to use of nonanhydrous $t-\mathrm{BuOH}$ or simply to atmospheric moisture. This is consistent with our earlier report that if water is excluded with molecular sieves, no aldehyde is observed. ${ }^{13}$ A lower catalyst loading of $1 \mathrm{~mol} \%$ may also be used to give the same TOF of $37 \mathrm{~h}^{-1}$ albeit at a slightly lower selectivity $(96 \%)$ and lower maximum aldehyde yield $(72 \%)$. The selectivity appears to be inversely proportional to catalyst loading, which is consistent with previous reports. ${ }^{6,16}$ Nevertheless, high aldehyde selectivity can still be achieved at high yields and catalyst turnovers, without the need for inert atmosphere or expensive reagents. The catalytic system is also tolerant of a variety of ring substituents such as alkyl, trifluoromethyl, ester, and nitro groups as well as halogens. Generally, the more electron deficient aromatic systems resulted in the highest yield of aldehydes. Electron rich systems such as $p$-tert-butylstyrene were less reactive under the reaction conditions, resulting in poorer yields.

(13) Dong, G.; Teo, P.; Wickens, Z. K.; Grubbs, R. H. Science 2011, 333,1609 .

(14) Mosciano, G. Perfum. Flavor. 1998, 23, 49.

(15) Chaintreau, A.; Joulain, D.; Marin, C.; Schmidt, C.-O.; Vey, M. J. Agric. Food Chem. 2003, 51, 6398.

(16) Feringa, B. L. J. Chem. Soc., Chem. Commun. 1986, 909.

(17) Ogura, T.; Kamimura, R.; Shiga, A.; Hosokawa, T. Bull. Chem. Soc. Jpn. 2005, 78, 1555.

(18) Yang, T.-K.; Shen, C.-Y. In Encyclopedia of Reagents for Organic Synthesis; Paquette, L., Ed.; J. Wiley \& Sons: New York, 2004.
Table 1. Styrene Oxidation Control Experiments ${ }^{a}$

\begin{tabular}{llccc}
\hline $\mathrm{BQ} / \%$ & solvent & $\mathrm{H}_{2} \mathrm{O} / \%$ & yield/\% & selectivity/\% \\
\hline 115 & $t$ - $\mathrm{BuOH}$ & 110 & 83 & 98 \\
115 & $i$-PrOH & 110 & 40 & 74 \\
0 & $t$ - $\mathrm{BuOH}$ & 110 & 5 & 100 \\
115 & $t-\mathrm{BuOH}$ & 0 & 38 & 95
\end{tabular}

${ }^{a}$ Reactions carried out in $0.125 \mathrm{M}$ solution $(0.1 \mathrm{mmol})$ with $2.5 \mathrm{~mol} \%$ catalyst loading at $85{ }^{\circ} \mathrm{C} .{ }^{b}$ Selectivity and yield determined by GC with tridecane as internal standard.

It was initially suspected that the conditions used for the hydration of styrene could be directly applied to the Wacker oxidation step by the removal of the reductants. However, without further modification beyond the removal of reductants, the product yield was much poorer, which we attributed to product instability and $\mathrm{CuCl}_{2}$ usage (vide infra). Using the original conditions for hydration $\left(10 \%\right.$ [Pd], $20 \% \mathrm{CuCl}_{2}, 100 \% \mathrm{BQ}, t-\mathrm{BuOH} / i-\mathrm{PrOH} 2: 1$, $0.0625 \mathrm{M}, 3 \mathrm{~h}$ ), only a $36 \%$ yield of aldehyde at $100 \%$ selectivity could be obtained ( $c f .85 \%$ anti-Markonikov products in hydration). ${ }^{13} \mathrm{~A}$ kinetic study for the oxidation of styrene was carried out, and it was observed that the product yield starts decreasing significantly upon reaching a maxima, likely due to aldehyde self-condensation (Figure 1). As such, all styrene oxidation reactions must be stopped once the product yield reaches its maximum to avoid product degradation. It is critical to note that when changing the scale of the reaction from $0.1 \mathrm{mmol}$ of styrene to $0.6 \mathrm{mmol}$ of styrene, we saw a notable delay in the reaction, leading to a shift in maximum yield from 45 to $60 \mathrm{~min}$ (see Supporting Information).

The usage of $\mathrm{CuCl}_{2}$ was also found to be unnecessary in the oxidation of styrene. In the presence of only $2.5 \mathrm{~mol} \%$ $\mathrm{CuCl}_{2}$, a $10 \%$ lower yield was obtained. This may be attributed to the increase in acidity of the reaction caused by $\mathrm{CuCl}_{2}$ which in turn results in product instability. The Wacker oxidation is also known to have an inverse secondorder dependence on chloride concentration, which likely contributes to decreased oxidation rates. ${ }^{19}$ Furthermore, lower catalyst loadings of $2.5 \mathrm{~mol} \%$ [Pd] could be used instead of the $10 \%$ required for hydration. Upon further reduction of the catalyst loading both anti-Markovnikov selectivity and yield are eroded.

As opposed to Feringa's $\mathrm{Pd} / \mathrm{Cu} / t-\mathrm{BuOH}$ system, for which a cyclic L-Pd- $\mathrm{NO}_{2}$ intermediate was proposed $\left(\mathrm{L}=\mathrm{MeCN}-\mathrm{CuCl}_{2}-\mathrm{Me}_{3} \mathrm{COH}\right)$ to account for antiMarkonikov selectivity, ${ }^{16}$ we postulate a tert-butyl vinyl ether intermediate (B) instead to give high regioselectivity. ${ }^{2}$ From earlier mechanistic studies, it was found that, in the presence of $t-\mathrm{BuOH}$, the olefin (A) would undergo Pdcatalyzed oxidation to generate $\mathbf{B}$. Due to the bulkiness of $t$ - $\mathrm{BuOH}$, the linear vinyl ether is preferred, which constitutes the key factor for high anti-Markonikov selectivity. During the Wacker process, acid is generated and, in the

(19) Keith, J. A.; Henry, P. M. Angew. Chem., Int. Ed. 2009, 48, 9038 9049 .

Org. Lett., Vol. 14, No. 13, 2012 
Table 2. Oxidation Reaction of Substrates $1-11^{a}$

$$
\underset{\begin{array}{l}
p \text {-benzoquinone }(115 \%) \\
\mathrm{H}_{2} \mathrm{O}\left(1 \text { equiv), } t-\mathrm{BuOH}, 85^{\circ} \mathrm{C}\right.
\end{array}}{\mathrm{PdCl}_{2}(\mathrm{MeCN})_{2}(2.5 \%)}
$$$$
\text { entry substrate } \quad \text { aldehyde (a) yield }{ }^{b} \quad \text { selectivity }^{c}
$$

\begin{tabular}{|c|c|c|}
\hline 1 & $\begin{array}{c}83 \\
\left(90^{d}\right)\end{array}$ & $\begin{array}{c}98 \\
\left(97^{d}\right)\end{array}$ \\
\hline 2 & 81 & 97 \\
\hline 3 & 90 & 96 \\
\hline 4 & 42 & 98 \\
\hline 5 & 96 & 99 \\
\hline 6 & 96 & $>99$ \\
\hline 7 & 74 & 99 \\
\hline 8 & 92 & 99 \\
\hline 9 & 90 & 99 \\
\hline 10 & 59 & 96 \\
\hline 11 & 72 & 99 \\
\hline
\end{tabular}

${ }^{a}$ Reactions carried out with $2.5 \mathrm{~mol} \%$ catalyst loading in $0.125 \mathrm{M}$ solution for $60 \mathrm{~min}$ at $85{ }^{\circ} \mathrm{C} .{ }^{b}$ Isolated aldehyde (a) yield (\%) (as 2,4dinitrophenylhydrazone derivative $(\mathbf{c})) .{ }^{c}(\mathbf{c}) /(\mathbf{c}+\mathbf{d})(\%) .{ }^{d} \mathrm{GC}$ yield and selectivity of aldehyde (a).

presence of water, the vinyl ether $\mathbf{B}$ is converted to the aldehyde $(\mathbf{C})$ via acid-catalyzed hydrolysis (Figure 2). ${ }^{13}$

In summary, a general and efficient method for the selective oxidation of aryl-substituted olefins to aldehydes has been described. This provides facile, direct access to a

(20) Kim, S.; Kim, D.; Parka, J. Adv. Synth. Catal. 2009, 351, 2573.

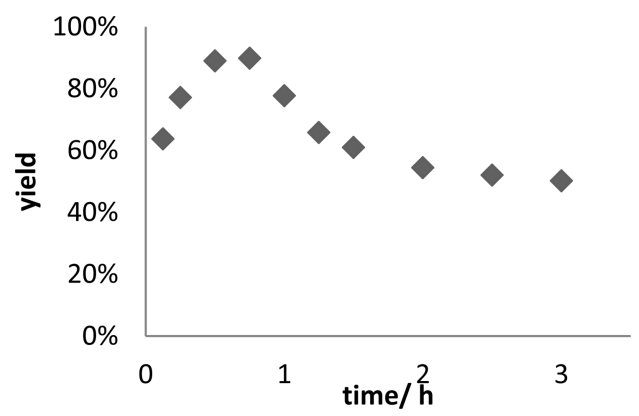

Figure 1. Aldehyde yield (\%) as a function of reaction time (h) for styrene Wacker on $0.1 \mathrm{mmol}$ scale.

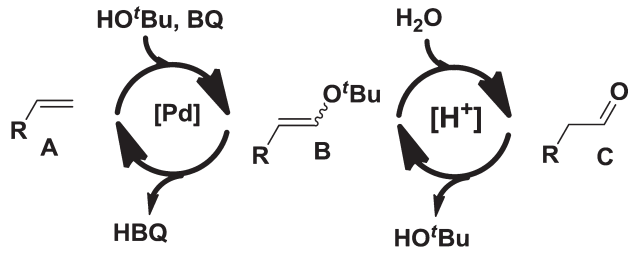

Figure 2. Proposed strategy for olefin oxidation.

previously challenging class of molecules. The current method shows a major improvement to that previously reported. ${ }^{16}$ Although oxygen cannot be used directly as the oxidant, hydroquinone can be readily recovered from the crude reaction mixture and converted to BQ in excellent yield via facile aerobic oxidation. ${ }^{13,20}$ Compared to methods for the Wacker oxidation currently in the literature, which typically give methyl ketones, the present method displays extraordinarily high aldehyde selectivity with high yields and turnovers. The simplicity of this method also makes it appealing for practical applications.

Acknowledgment. We gratefully acknowledge financial support from King Abdullah University of Science and Technology Centre in Development, King Fahd University of Petroleum and Minerals, and the NSF. G.D. thanks the Camille and Henry Dreyfus Foundation for a postdoctoral fellowship.

Supporting Information Available. Detailed experimental procedures and analytical data supplied as Supporting Information. This material is available free of charge via the Internet at http://pubs.acs.org.

The authors declare no competing financial interest. 